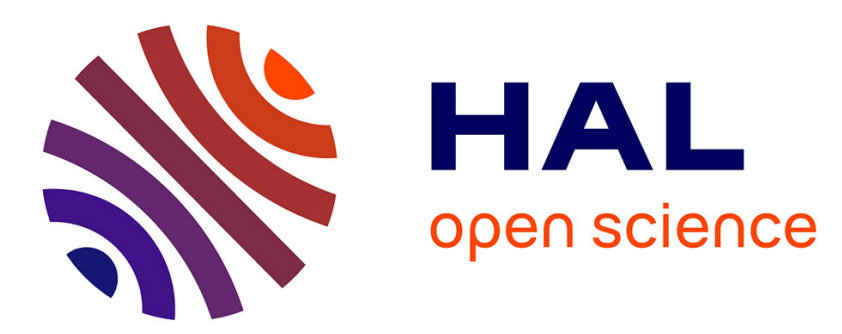

\title{
La Méditerranée et le voyage dans le Florimont en prose
} Marie Madeleine Castellani

\section{To cite this version:}

Marie Madeleine Castellani. La Méditerranée et le voyage dans le Florimont en prose. Écrire le voyage au temps des ducs de Bourgogne (Dunkerque) Écrire le voyage au temps des ducs de Bourgogne, Jean Devaux, Matthieu Machal, Alexandra Velissariou, Oct 2017, Dunkerque, France. hal-01728361

\section{HAL Id: hal-01728361 \\ https://hal.univ-lille.fr/hal-01728361}

Submitted on 10 Mar 2018

HAL is a multi-disciplinary open access archive for the deposit and dissemination of scientific research documents, whether they are published or not. The documents may come from teaching and research institutions in France or abroad, or from public or private research centers.
L'archive ouverte pluridisciplinaire HAL, est destinée au dépôt et à la diffusion de documents scientifiques de niveau recherche, publiés ou non, émanant des établissements d'enseignement et de recherche français ou étrangers, des laboratoires publics ou privés. 
La Méditerranée et le voyage dans le Florimont en prose

Le voyage en Méditerranée dans le Florimont en prose"

Dans le Florimont en prose, réécriture du Florimont d'Aymon de Varennes ${ }^{1}$, la Méditerranée joue un rôle central, tant dans la version d'origine que dans la mise en prose bourguignonne ${ }^{2}$. Cela n'a rien d'étonnant et était souligné par Danielle Quéruel :

$\mathrm{Au} \mathrm{XV}$ siècle les romans de chevalerie - qui se font nombreux - continuent à privilégier ce cadre narratif qui se dessine autour de la Méditerranée et ont le plus souvent pour point commun de mettre en scène des personnages qui se partagent entre deux mondes : l'Occident où ils sont nés et les royaumes d'outre-mer où leur destin les conduit ${ }^{3}$.

Mais le Roman de Florimont se distingue en plusieurs points des autres biographies chevaleresques du fonds Wavrin. Tout d'abord, le récit se situe dans l'Antiquité puisque le héros est un ancêtre d'Alexandre. C'est pourquoi les voyages en mer se font sous la protection de Neptune : Ilz reclamerent Neptunus, le dieu de la mer (VI, 16) et sy se commanderent au dieu Neptunus (CLXI, 11). D'autre part, le parcours du héros ne peut, pour des raisons évidentes, passer par la croisade. Certes, le pèlerinage à Jérusalem est bien mentionné dans le prologue. Alors qu'Aymon n'expliquait pas sa présence en Grèce, à Phelipople 4 où il aurait trouvé le texte de sa source (v. 31-34), le prosateur place son départ en orient sous le signe du saint voiage d'oultremer vers la sainte chitey de Jherusalem (Pr., I). Or, ce Picard et ses compagnons des païs de Borgongne et d'ailleurs vont être détournés de ce projet initial. Dieu, la mer et le vent les conduisent en la chité de Sallenicque (Pr., 2-3). La puissance divine détourne les voyageurs du droit chemin de leur pieux projet pour les diriger rapidement vers la mer Égée (Archipelago) et à Salonique, vers la découverte du texte :

Il covint par force que nostre galee sur coy estiemmes tournast hors de nostre droit chemin et fusmes constraint de abandonner a Dieu, a la mer et au vent la conduitte et garde de nous tous et tans que en pau de temps, passames au travers de 1'Archepelago. (Pr., 3)

\footnotetext{
${ }^{1}$ Cf. Aimon VON VARENNES, Florimont, éd. A. HILKA, Göttingen, 1932 (Gesellschaft für romanische Literatur 48), qui suit principalement $F$ (BnF fr. $15101 F^{l}$ et $F^{2}$ ).

${ }^{2}$ Que l'on citera dans l'édition d'Hélène Bidaux.

3 «Pourquoi partir? Une typologie des voyages dans quelques romans de la fin du Moyen Âge», Guerres, voyages et quêtes au Moyen Âge, Mélanges offerts à Jean-Claude Faucon, études réunies par Alain Labbé, Daniel W. Lacroix et Danielle Quéruel, Paris, Honoré Champion, 2000, p. 333-348, citation p. 333.

${ }^{4}$ Philippopolis de Thrace, aujourd'hui Plovdiv en Bulgarie, prise en 342 par Philippe, père d'Alexandre, appartient bien historiquement à l'histoire de la Macédoine.
} 
Point de Terre sainte donc, mais une description des territoires que va conquérir le premier Philippe, autour de l'Hellespont. L'énumération des territoires vient de la source :

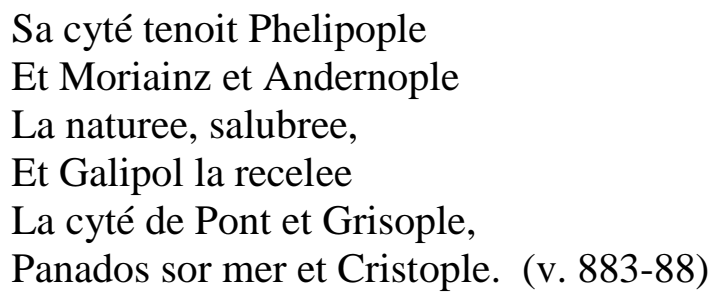

Autour de 1'Hellespont (le Pont-Euxin, la mer noire ${ }^{5}$ ), ici assimilé à une ville (La cyté de Pont), on trouve la cité fondée par Philippe, Philipopolis en Thrace (aujourd'hui Plovdiv en Bulgarie), Maronia (en face de l'île de Samothrace), Andrinople ${ }^{6}$, Galipoli (Gallipoli aujourd'hui), Chrysopolis, aujourd'hui Üsküdar, un des trente-neuf districts de la ville d'Istanbul, Panados, sur la mer de Marmara ${ }^{7}$ (Panium, sur la côte du Bosphore de Thrace), et Christopolis (aujourd'hui Kavala ou Kavalla, dont Christoupolis est le nom byzantin). La prose donne ici moins de noms : selon elle, Philippe, ayant laissé à son frère Madian le royame d'Egipte et d'Arrabe, devient Sires [...] d'Andernoble, de Gallippoly, de Sallenicque, de Negrepont, de Bisance, qui maintenant se nomme Constantinoble, et de moult aultres grans contrees (IX 13). Au texte d'Aymon, la prose ajoute Salonique et modernise l'ancienne Byzance. Les villes retenues sont sur les frontières de l'empire d'orient, qu'il s'agisse d'Andrinople entre la Grèce et Bulgarie, de Gallipoli, la péninsule antique de la Chersonèse de Thrace $^{8}$ ou en constituent le cour : l'Hellespont, Constantinople et la ville où la volonté divine conduit le voyageur, Salonique ${ }^{9}$, enjeu de conquête tout au cours du $\mathrm{XV}^{\mathrm{e}}$ siècle entre les Grecs de Constantinople et les Ottomans. À la date indiquée par le narrateur au début du texte, 1418, Salonique, ancienne capitale du royaume, fait toujours partie de l'empire et cela depuis le premier quart du XIV ${ }^{\mathrm{e}}$ siècle $(1313)^{10}$.

Ce resserrement témoigne donc d'une conscience des menaces qui pèsent sur l'empire chrétien d'orient. Le narrateur souligne dans son prologue l'ancrage chrétien de cet empire, en

\footnotetext{
${ }^{5}$ Situé sur la rive orientale du Bosphore (où l'on trouve la tour de Léandre, souvenir de l'histoire d'Héro et Léandre et de la noyade du jeune homme

${ }^{6}$ L'ancienne Andrinople est aujourd'hui Édirne, préfecture de la province turque du même nom, limitrophe de la Bulgarie et de la Grèce. La ville compte environ 143000 habitants. Elle est traversée par la Maritsa qui passe aussi en Bulgarie.

${ }^{7}$ PANIUM, (Géog. anc.), promontoire d'Europe, sur la côte du Bosphore de Thrace, parallèle, selon Pierre Gilles, aux îles Cyanées.

${ }^{8}$ En Turquie actuelle.

${ }^{9}$ Depuis la fin du XIV ${ }^{\mathrm{e}}$ siècle, Grecs et Ottomans se disputent la ville qui tombera en 1430 sous contrôle ottoman.

${ }^{10}$ Redevenue ottomane en 1430, ayant été reprise à Venise.
} 
montrant les voyageurs faisant leurs dévotions à Salonique : devotement venismes rendre graces en l'eglise Saint Pol ${ }^{11}$ a nostre Seigneur et a la Virge Marie sa mere (Prologue 3). Au passage, le narrateur fait l'éloge des coustumes et merveilles du païs de Grece (Pr., 4) et de la chevalerie grigoise; on pourrait y voir un conseil à Philippe le Bon (destinataire de l'ouvrage) qui, selon J. Heers ${ }^{12}$, «ne songeait pas à porter secours aux Grecs de Constantinople mais à délivrer Jérusalem », à être plus attentif aux dangers courus par l'empire d'orient face à la menace turque. La mention de Nicopolis sur la partie terrestre du chemin du retour lors de l'épisode carthaginois rappelle à la mémoire le terrible désastre subi par les armées d'occident engagées dans la croisade et alliées au roi de Hongrie Sigismond ${ }^{13}$ :

16 Sur leurs chevaulx cheminerent tant qu'ilz ariverent a Nicolpoly quy siet sur la Dunoe ; la passerent puis entrerent en Hongrye ou a grant joie et [fol 231r] leesse furent recheu. 17 Atant vous lairay a parler d'eulx et tourneray a nostre droitte matere. $(198,14-17)$

Mais il en exorcise aussi le souvenir car ce nom apparaît lors du retour triomphal du héros d'une expédition réussie grâce, entre autres, à l'alliance avec un roi de Hongrie, Candiobras, dont le héros a auparavant gagné l'amitié après l'avoir victorieusement combattu et avoir conquis sur lui des terres que Grecs et Latins d'orient disputent alors aux Turcs ${ }^{14}$. Les voisins menaçants de Philippe, le Hongrois Candiobras et ses alliés bulgares, évoquent ceux qui depuis plusieurs siècles menacent Constantinople; certains des empereurs ont essayé de la contrecarrer par des alliances matrimoniales ${ }^{15}$, ce dont la volonté de Candiobras d'épouser la fille de Philippe pour s'emparer ses terres se fait l'écho. Candiobras a aussi pour allié le roi de Valachie, pays que traversent ses ambassadeurs pour défier Philippe :

Sy se misrent a point et prindrent congiet du roy et de la baronnye et tindrent leur chemin par la Valacquie*, puis entrerent en la Dunoe, puis vindrent a Flourentin en Boulgarye et firent tant par leurs journeez qu'ilz ariverent en la Gresse et vindrent tout droit* a Philippople ${ }^{16}$. (XIV, 3)

\footnotetext{
${ }^{11}$ On sait que saint Paul a adressé depuis Corinthe deux de ses épîtres aux habitants de la région $\left(1^{\mathrm{re}}\right.$ et $2^{\mathrm{e}}$ Épitres aux Thessaloniciens).

${ }^{12}$ J. HEERS, Chute et mort de Constantinople (1204-1453), Paris, Perrin, 2005, p. 230. En 1421, Gillebert de Lannoy ne s'arrête pas Constantinople, lui préférant la Syrie et l'Égypte (Ibid., p. 230-31). ${ }^{13}$ Le 25 septembre 1396.

${ }^{14}$ Chez Aymon, le sénéchal Damien conseille à Philippe de faire appel au duc d'Albanie, au roi d'Esclavonie, au roi de Perse et à ses alliés italiens (Pouille, Toscane, Romagne).

${ }^{15}$ Dès le XIII ${ }^{\mathrm{e}}$ siècle, Michel Paléologue « assurait ses positions en Orient en promettant sa fille Marie au roi de Bulgarie et son fils Andronic à Anne, une des filles du roi de Hongrie. », J. HEERS, op. cit., p. 193.

${ }^{16}$ Je n'ai pas pu identifier pour l'instant Flourentin.
} 
Longée au sud par le Danube (la Dunoe) la Valachie, une des provinces de l'actuelle Roumanie $^{17}$, était depuis novembre 1330 et la bataille de Posada une dépendance de la Hongrie: l'alliance entre les deux pays, le roi de Valachie combattant aux côtés de Candiobras (ch. 140) avant d'être fait prisonnier par Florimont (ch. 141) correspond donc bien à la réalité du temps.

Une fois soulignées ces différences importantes avec la source, dont certaines viennent d'une prise en compte de l'actualité contemporaine à la mise en prose, il n'en reste pas moins que, parmi les romans de l'atelier Wavrin, c'est sans doute le Florimont qui compte le plus grand nombre de déplacements, voyages ou d'expéditions militaires dans des territoires éloignés, surtout maritimes, mais aussi terrestres on l'a vu, et cela grâce au fait que le roman propose une double biographie chevaleresque, celle de Philippe de Macédoine et celle de Florimont de Duras, qui, au contraire des héros évoqués plus haut, appartiennent par leur origine aux deux espaces méditerranéens, oriental et occidental.

Le parcours initial de Philippe ${ }^{18}$ balaye toute l'étendue de l'orient, du Caire (Babilonne) et du port de Damiette à Patras (ch. IV-VI), avant de se poursuivre vers l'Hellespont par les terres. La prose modifie ici le voyage terrestre du jeune Philippe. Les vers suggèrent en effet qu'il suit depuis l'Egypte une route terrestre par la Syrie, Antioche et la Turquie avant le passage du Bras saint Georges (l'Hellespont), pour arriver à Avedon/Boucadaïde (v. 445-46), c'est-à-dire Abydos sur l'Hellespont. Il fait ainsi, à l'envers, la route des croisés occidentaux. Dans la prose Philippe, parti de Damiette, arrive, malgré une traversée difficile et une tempête qui disperse les navires, à Patras, bien identifiée par le narrateur comme une ville connue de son temps : qui se nommoit a celluy temps Patras et encores fait $(\mathrm{V}, 18)$. De nouveau l'accent est porté sur un parcours qui mène vers l'Hellespont en venant de l'ouest et un rôle important est donné à Patras, port tourné vers la Méditerranée occidentale, notamment l'Albanie. Le déplacement terrestre crée une profondeur mémorielle, en évoquant la Thèbes antique, mais contient aussi un avertissement sur le caractère fragile des empires qui fait lui aussi écho aux menaces présentes :

puis s'en party le roy et se mist a chemin. 26 Il passa par la place ou jadis ot esté la grant citey de Thebes. 27 Moult fort la regarda et vey la grant ruyne qui bien demonstroit que grant chose avoit esté*. 28 Il passa oultre par Thesaille, sy

\footnotetext{
${ }^{17}$ Bucarest en fait partie.

${ }^{18}$ Florimont, v. $120-1672$; mise en prose, f. $2^{\mathrm{r}}-26^{\mathrm{r}}$, ch. II-XVIII.
} 
chemina par forestz et par landes et tant que, d'aventure, prist son chemin vers la Dunoe. 29 Il choisy le païs moult bel et plain ou estoient foison prés, bois, rivieres qui moult luy pleurent et disoit que oncques nul jour plus bel païs n'avoit veu et marchissoit la contree au païs d'Albanye (VI, 25-29)

L'Albanie est le pays natal de Florimont, qui vient de Duras (Durazzo ${ }^{19}$ ) en Méditerranée occidentale; c'est un port qui sert de cadre à plusieurs scènes tant pour les principaux personnages que pour des silhouettes plus secondaires : c'est là qu'accostent les messagers envoyés par Garganeüs réclamer tribut ou encore, au chapitre LVII, le prince Rissus, seigneur de Calabre et de toute la terre de Labour ${ }^{20}$ (la Campanie), qui règne sur une cité qui sur le Far est assise laquelle se nomme Regis ${ }^{21}$, Reggio de Calabre sur le détroit de Messine ${ }^{22}$. Le territoire du géant Garganeüs se situe chez Aymon an Puille (v. 3095) et son château dans un plain/Entre Sipon[t] e Mon[t] Gargain (v. 3097-98). La prose reprend cette localisation, y ajoutant la terre de Raguise (Raguse, l'actuelle Doubrovnik en Bosnie ${ }^{23}$ ). Celle-ci sera évoquée à deux reprises, notamment à la fin de l'épisode carthaginois, où elle est la ville du roi de Hongrie, désormais allié du héros, ce qui permet d'y faire étape sans difficulté dans le long voyage menant de Carthage en Grèce :

14 Firent voile et singlerent tant par mer au bon vent qu'ilz orrent que ilz passerent le goulfe de Lyon et les isles, tant qu'ilz vindrent vers Courfo, sy entrerent en la mer Adriane et prindrent port a Raguise, quy pour le temps estoit au roy de Hongrye. $15 \mathrm{Ilz}$ saillirent des nefz et se rafressirent huit jours entiers en la citey ; au neuvieme se deppa[r]tirent* par terre.

L'Adriatique sert de cadre aux premières aventures de Florimont : lutte contre le monstre marin et contre le géant Garganeüs, qui habite les Pouilles, face à l'Albanie du Monte Gargano $^{24}$, amours avec la fée de l'île Celée ${ }^{25}$. Commençant la construction de ce qui sera un

\footnotetext{
${ }^{19}$ Duras est le nom français médiéval de l'actuelle Durrës, deuxième ville d'Albanie, l'ancienne Epidamne ou Dyrrachium.

${ }^{20}$ La Terre de Labour (Terra di Lavoro), dont le nom vient des Liburi, désigne la "Campanie heureuse » dont la ville principale est Capoue. C'est, avec l'Apulie et la Calabre, l'une des trois provinces normandes de Sicile. Faire de Rissus un prince de Calabre et de Labour correspond donc à une réalité historique.

${ }^{21}$ Le nom proposé est proche de Righi en Grec de Calabre, P

${ }^{22}$ Et non l'estuaire du Tibre, comme dans La Manekine. À l'époque du récit, ces territoires appartiennent au royaume d'Anjou-Sicile.

${ }^{23} \mathrm{Ch}$. XXI, 5 Il estoit yssus des jayans qui par avant soloyent estre environ la terre de Raguise, [fol $30 v]$ qui en ce temps estoit a Garganeüs.

${ }^{24}$ Le Gargano, à l'origine une île, rattachée par la suite à la terre ferme, correspond au massif montagneux appelé sperone d'Italia qui se projette dans la mer Adriatique et délimite le golfe de Manfredonia. Vers l'est, il s'étend jusqu'au cap de la Testa del Gargano.
} 
vaste empire, il réunit à la Macédoine par son mariage, l'Albanie et ses États alliés (la Calabre de Rissus et l'Esclavonie de Médon), avant d'aider son beau-père à conquérir Bulgarie et Hongrie. Quant à Chyprienne, la nourrice de son épouse, la jeune Rommadanaple, elle estoit natisve de Nicosye en Chypre, l'un des grands comptoirs permettant de passer d'Occident en Orient, de faire étape en Méditerranée, notamment sur le chemin de la Terre sainte, appartenant encore en 1418 à la date du texte, aux Lusignan. L'enjeu politique que constitue la conquête des territoires c'est aussi avoir des portes sur la mer qui permettent de contrôler les déplacements. C'est ce qu'explique Florimont à son oncle, Medon, le roi d'Istrie ${ }^{26}$, en conflit avec Amadas (ch. XXXIV-XXXV) :

Sire, ce dist Flourimont, puisque de ceste chose me faittes juge, je voel que le roy Amadas qui cy est, deviengne [ fol 55r] vos homs et que de vous reprengne sa terre et tout son royaume, et tous les barons de ses paÿs vous fachent hommage; et avoec ce, que les deux pors de mer qui sont en son royaume, c'est assavoir Parete et Rouvingne, soyent mis en vostre main, esquelz deux villes porrés mettre vos gens et officiers de par vous. » $(\mathrm{XL}, 11)$.

Pour l'expédition de Florimont vers l'Afrique du nord et Clavegris, afin de délivrer le père de Florimont prisonnier de l'émir de Carthage, la prose seule explique aux voyageurs, par la voix de Maître Flocart, quel chemin ils devront suivre depuis Philippopolis jusqu'à Patras, puis par mer jusqu'à l'Afrique du nord, sans apparemment s'arrêter en chemin, ce qui constitue un long voyage, sans doute quand même peu éloigné des côtes grâce aux nombreuses îles qui jalonnent le parcours :

$9 \mathrm{Ilz}$ se partirent de Philippople; tant esrerent que a Patras ariverent ou la navire fu toutte* apprestee. 10 Plus estoient de deux cent mille homes, qui tous entrerent dedans les nefz. 11 Apprés ce leverent les ancres quant tous furent entré dedans, puis firent voile, sy se commanderent au dieu Neptunus et nagerent tant et singlerent que tout droit vers Clavegris s'adrecherent. $12 \mathrm{Ilz}$ passerent touttes les isles qui en la Boucque de Lyon ${ }^{27}$ sont, c'est assavoir Sesisle ${ }^{28}$, Corsegne, Sardaigne, Maiorque et Manorque et Evisse [Ibiza]. 13 Tant singlerent de nuit et de jour que au chief de l'isle de Magalon [fol 185v] ariverent. (Ch. 161, 7-13)

\footnotetext{
${ }^{25}$ La situation de cette île merveilleuse n'est pas donnée et la mère de Florimont surprend sans doute la fée avec son fils non loin de Duras ; des indices pourraient permettre de situer l'île en question dans la Méditerranée orientale, su côté de l'Archipelago.

${ }^{26}$ L'Istrie est une péninsule de l'Adriatique de forme triangulaire pointée vers le sud, attachée au continent par le nord-est, un territoire enfoncé dans la mer et comportant des îles.

${ }^{27}$ On serait tenté d'y voir Le golfe du Lion, mais cela ne convient pas bien géographiquement. À noter qu'il existait à Venise une « bouche du lion », où l'on pouvait glisser des dénonciations anonymes.

${ }^{28}$ Et non les isle [s] comme il est indiqué dans la transcription d'H. Bidaux.
} 
Cet ajout témoigne d'une bonne connaissance de la géographie de la Méditerranée occidentale régions perçues comme sarrasines - Solimant et son frère ont été affolés ${ }^{29}$, c'est-à-dire castrés pour garder la femme de l'émir - et les Carthaginois seront plus loin présentés comme cruels ou présomptueux. Les rois de Barbarie, de Crète, de Rhodes et de Chypre, d'abord ennemis des Macédoniens, participent à cette expédition. Déjà vaste ${ }^{30}$ chez Aymon, cette alliance est augmentée de quelques nouveau membres dont le roy de Valacquie $(189,18)^{31}$, un ancien adversaire, et le prince Rissus, un ami fidèle depuis son arrivée à Duras.

À travers les déplacements du héros, lui-même fleur de cette chevalerye grigoise qui jadis fu moult hault eslevee par tous rengnes (Pr., 4), se dessine le rêve d'une Méditerranée unie de l'occident à l'orient sous la houlette d'un prince idéal. Le Roman de Florimont fait procéder son héros à une conquête systématique de l'espace méditerranéen tout entier, soit en s'alliant des adversaires par ses qualités, soit en détruisant des ennemis jugés irréductibles, qu'il s'agisse de monstres comme Garganeüs, yssus des jayans qui par avant soloyent estre environ la terre de Raguise et le promontoire du Monte Gargano ${ }^{32}$ ou de peuples présentés comme Sarrasins, comme à Clavegris ; contre eux Florimont déploie tout autant la force physique que la ruse, souvent associée à l'usage de la mer et des bateaux : ainsi contre Garganeüs, il cache ses hommes dans le navire :

Flourimont dist a sa gent : 12 «Seigneur, je vous prye que nulz de vous ne s'amonstre car point ne voel que soiés veus ne apparcheus. »13 Tous respondirent que son commandement ilz feroyent. $14 \mathrm{Ilz}$ furent en la nef sy coy et sy taisant qu'il sambloit que dedans n'euist personne, fors seulement Flourimont et les

\footnotetext{
${ }^{29}$ Cette variante ne se trouve pas dans toutes les versions en vers (le manuscrit retenu par Hilka dit simplement que l'émir a fait deseriter (v. 12153) les deux frères); afoler est cependant donné par un nombre important de mss (ABEGH ${ }^{2}$ IKLT). On a déjà noté la fréquente conformité de $\mathrm{H}^{2}$ avec notre prose.

${ }^{30}$ Chez Aymon, les alliés sont Afrique, Barbarie, la Turquie devers le Coine (c'est-à-dire le sultanat d'Iconium), Crète et Couloine (Cologne), la terre de Rousie, Hongrie, le roi Medon d'Esclabonie, oncle de Florimont ainsi que les gens de Babilonne (le frère aîné de Philippe), venus de haute mer (v. 11805-17).

3116 Alors, quant le roy ot entendu la guette, il ordonna ses gens en quatre battailles : la premiere bailla a conduire $\mathbf{a}[\mathbf{u}]$ roy de Hongrie, en laquelle estoient trente mille hommes; avoec luy avoit le roy del Coine. $17 \mathrm{La}$ deuxieme battaille ordonna au roy de Crette, en laquelle avoit aultres trente mille [fol 217v] homes. $18 \mathrm{La}$ terche battaille bailla a conduire au roy de la Valacquie, qui pareillement avoit trente mille homez fors et hardis pour maintenir estour ; et la quarte battaille le roy Flourimont conduisy a tout cinquante mille tresexpers et vaillans homez quy grant voloir orrent de trouver leurs anemis. 19 Le roy Flourimont avoit avoec luy son biau pere, le roy de Barbarye, et son oncle, le roy d'Esclavonnye*. 20 Le noble prinche Rissus y fu, le duc d'Anternoble et Damyen le seneschal. (Ch. 189

${ }^{32}$ Le Monte Gargano (Mont Gargain dans les textes français) important promontoire de la péninsule italienne qui s'avance dans la mer Adriatique, lui valant le surnom d'éperon de l'Italie.
} 


\section{maronnyers qui tant firent que lor nef misrent droit au port ou ilz ancrerent.}

ou encore dans l'épisode de Clavegris, il déguise ses hommes en marchands.

$* * *$

Du fait de la présence de la mer, du rôle qu'elle joue dans les déplacements et les conquêtes du héros les navigations scandent le texte. On nous donne quelques distances, en durée de voyage, par exemple six jours de mer paisible pour mener la future femme de Philippe de « Tripoli en Barbarie » à Patras, d'ouest en est

22 Pour le bon temps qu'ilz orrent, leverent les ancres, sy firent voile et orrent sy bon vent que en six jours furent arivez a Patras, la ou a moult grant joye et solempnité furent recheus (XI, 21-22)

Entre l'Égypte et Patras à deux jours de voyage sans encombres succèdent quatorze jours de tempête, sans pouvoir accoster. Après la victoire sur Garganeüs c'est un bon vent qui en pou d'eure mène le héros et ses compagnons jusqu'à Duras (ch. XLIX, 34), cette bonne volonté des éléments s'accordant à l'euphorie qui règne parmi les compagnons de Florimont victorieux de Garganeüs :

Puis tost apprés se departy Flourimont et s'en revint a grant joye et leesse, luy et ses gens, entrer dedans la nef. 33 Quant tous furent entré dedans, ilz leverent les voilles. $34 \mathrm{Ilz}$ orrent bon vent qui en pou d'eure les mena au port de Duras, la ou a moult grant joye furent recheu du duc Matacart et de la ducesse Ydorye, qui saouler ne se pot de baisier et conjoÿr leur filz.

Le texte offre plusieurs termes désignant les bateaux: navire(s)(plus d'une trentaine d'occurrences), pour les bateaux ou comme terme générique désignant la flotte, nefs, de loin le plus fréquent (une bonne cinquantaine d'occurrences de nef, nefs et nefz) qui peuvent être richement apareillies, et dont on lève l'ancre pour partir (VI, 14), galees (5 occurrences : XLII, 10 (pillées par les pirates), CLXXXVIII, 3 et 8 ; la navire et les cinquante galees touttes armees et garnyes de tout ce que mestier estoit 189, 4 ; 191, 27), batel (3 occurrences : XLIV, 6, XLVI, 6 et 15), comme barge(s) (IV, $18 ; 159,5 ; 185,3)$. Quatre passages seulement (VI, 6 et 12 : XI, 21, 190,11) évoquent les marins, marroniers moult desyrant de partir (XI, 21); les bateaux esploit [...] ent au vent et au voile (XLIV, 6) pour quitter le port ou ariver à destination (XLVII, 2), les nefs font mouvement sous le vent, comme la belle nef qui a plain voyle venoit ancrer au port de Duras, LVII qui conduit le prince Rissus. Deux passages sont plus développés, le départ de Florimont pour combattre Garganeüs, ce qui donnera également lieu, on l'a vu, à une ruse du héros : 
et quant ce vint le lendemain matin, Flourimont, qui moult desiroit de son emprise mettre a fin, fist son harnas trousser et porter dedans sa nef, laquelle estoit grande et large. 4 Moult bien le fist garnir de vivres et de tout ce que mestier luy estoit. 5 Il fist ses gens et ses chevaulx entrer dedans, puis vint prendre congiet au duc son pere et a Idorye sa mere, qui en plourant le baisa moult de fois. 6 Apprés prist congiet des bourgois de la citey, sy s'en vint entrer dedans son batel, qui desja estoit prest et appareilliés pour lever les ancres. (46, 3-6)

Le motif est ici à peu près complet : préparatifs de départ, vivres, adieux pleins de tristesse, départ par les ancres levées, sans mention cependant des marins. Un autre passage, qui réunit le plus grand nombre de termes différents désignant les bateaux décrit là encore les préparatifs d'une des expéditions militaires, notamment les vivres nécessaires au voyage :

5 Le roy Flourimont, qui moult grant desir ot de ceste oevre achever et mettre a fin, fist envoier gens par tous les pors de Grece prendre et retenir les vaissiaulx, nefs, barge[s] et dromons ; garnir les fist de pain, de bestail, de vins, de chars, de poissons et de tout ce qu'il convenoit et qui estoit appertenant sur navire pour faire guerre. 6 Il les fist touttes venir et dessendre devant Patras, et quant touttes les nefz furent a Patras assamblees et venues, ilz furent bien cent vingt* nefs bien garnies et ordonnees. (CLIX, 5-6)

Mais le plus souvent ces préparatifs et les manœuvres de départ sont décrits de façon bien plus brève, par exemple lors du voyage vers Clavegris ${ }^{33}$ :

a Patras ariverent ou la navire fu toutte* apprestee. 10 Plus estoient de deux cent mille homes, qui tous entrerent dedans les nefz. 11 Apprés ce leverent les ancres quant tous furent entré dedans, puis firent voile, sy se commanderent au dieu Neptunus et nagerent tant et singlerent que tout droit vers Clavegris s'adrecherent.ch. 161

au point qu'Aymon lui-même est parfois plus précis dans ses descriptions de la navigation, ainsi pour le départ d'Egypte :

Li maronier orent bon vent 386

Li airs fu clercs et boens li vans 391

Les neis flotoient sor la mer

Il corrent les ancres lever,

Les voiles desserent a vent,

Si governerent durement,

Le jor corrent a plaingnes voiles,

La nuit asiment as estoiles $427-432$

\footnotetext{
${ }^{33}$ Il peut même arriver que le voyage soit totalement éludé : 14 Des adventures ne des fortunes qu'il pooit avoir en chemin, ceste histoire n'en fait nulle mencyon, ains fist tant que luy et sa compaignye, raemplye de grans richesses que ilz orrent gaigniet ou voiage, ariverent ens* ou port de Salenicque. $(205,14)$
} 
La version en prose dit simplement : firent voille ; en revanche, elle ajoute une invocation à Neptune, nécessaire car la mer apparaît comme le lieu du danger et de la tempête : dès cette première traversée entre Égypte et Grèce (chapitre VI), les navires sont soumis à la tempête et aux caprices des vents ${ }^{34}$; la fortune de mer, terme spécifique à la prose, hante les imaginaires et la tempête peut durer de longues journées, ici quatorze jours (la même durée que dans les vers) qui séparent les groupes de navires et qui laissent les voyageurs mout mat et travilliet de la tempeste de mer (ch. VI, 19) et las leurs chevaux, qui font la traversée avec leurs cavaliers, habitude également évoquée lors de l'arrivée de Rissus à Duras :

le Povre Perdu regarda sur la mer et perchupt une belle nef qui a plain voyle venoit ancrer ou port de Duras. 5 Quant assés elle fu prés*, il oÿ hennir chevaulx qui en la nef estoyent, dont en son ceur s'ejoÿssoit car moult volemptiers les oÿ. 6 La nef estoit du paÿs de Calabre. 7 Dedans fu ungs prinches quy Rissus ot a non. 8 Mandé l'avoit le roy Philippe de Machedonne. (LXVII, 2-7)

Plus que la tempête elle-même, le texte en évoque les effets sur les voyageurs (moult mat et travilliet VI, 19) notamment la peur : bien cuiderent tous perir et noyer. Cette crainte de la noyade, qui hante les esprits puisqu'on ne peut être enseveli, est présente dans tout voyage en mer, d'où les deux motifs opposés de la tristesse des départs et de la joie des retours, par exemple lorsque les compagnons de Florimont entrent dans la nef qui va les conduire vers Garganeüs : 7 Tout en plourant, ses gens y entrerent, car bien cuidoyent que jamais n'en deuissent retourner. En revanche, au retour les termes signifiant la joie se multiplient, comme on a pu le voir plus haut. La crainte de la mer est telle qu'elle peut même faire naître des monstres imaginaires comme dans le rêve du duc Matakart :

13 sy sambla au duc qu'il regardoit vers le port de mer ou il vey une nef ariver, dont dehors sailloit ungs monstres qui de pluiseurs levriers estoit acompaingniés, en laquelle compaignye le lionchel se tient tant que las et moult deffais s'en retourna vers le duc (XIX, 13-14).

Elle est par excellence le lieu le plus susceptible d'engendrer des monstres, comme celui que combat Florimont et qui retrouve des forces en allant se baigner. La double menace du monstre marin ${ }^{35}$ et du géant du promontoire du Monte Gargano fait de l'Albanie une terre

\footnotetext{
${ }^{34}$ On opposera la tempête du prologue au bon vent qui en pou d'eure les mena au port de Duras au ch. XLIX, 34, après la victoire sur Garganeüs.

358 D'aultre part, y avoit ung monstre qui moult grant guerre luy faisoit et destruisoit sa terre. 9 Celuy monstre conversoit et abittoit sur la rive de la mer; moult estoit mervilleux a voir. 10 Il ot chief de
} 
gaste, que seule l'action du héros civilisateur pourra restaurer grâce à un déplacement maritime qui le fera s'affronter au danger. Ce sont ses liens avec la mer qui rendent dangereuse la fée de l'île Celée, dont l'origine même la relie à la mer $^{36}$, parce qu'elle veut entraîner le héros hors du monde de la chevalerie, mais aussi parce qu'une fois abandonnée par lui, elle épouse Neufas, neveu de Candiobras, et donne naissance au magicien Neptabus (Nectanabus), qui par ses enchantemens faisoit les nefs plongier et perir en la mer. (LII, 30) ${ }^{37}$. La piraterie apparaît comme l'un des grands dangers des voyages maritimes. C'est ce que rappelle Danielle Quéruel :

Les traversées en mer sont toujours inquiétantes : on doit redouter tout d'abord les tempêtes qui dévient les bateaux vers des côtes inhospitalières, mais aussi les rencontres avec des nefs ennemies, chargées de Sarrasins ou de pirates. [...] Sans doute faut-il percevoir ici l'écho de peurs véritables, mais qui sont systématiquement récupérées par matière romanesque ${ }^{38}$.

Notre roman en fait aussi foi, en reportant cependant le motif des pirates sur des personnages secondaires : ceux qui enlèvent la mère de Garganeüs, fille de l'émir de Carthage destinée à un roi de Lybie, Neptabus, le fils de la fée, enfin Garganeüs lui-même, pillard qui ravage les îles alentour :

[...] n'a nulles isles en ceste mer que par ses galees ne soyent pillees et robees, car, se aulcuns en y a qui a l'encontre de luy se rebellent, et que au dessus en puist venir, moult en prent grant pugnicion, car morir miserablement et a grant destresse les fait en ses prisons, par rage de fain, ne jamais raenchon n'en voelt prendre. (XLVV, 10)

Les dangers maritimes sont plutôt utilisés dans la narration une perspective dramatique : l'enlèvement de la mère de Garganeüs provoque par vengeance celui du père de Florimont et devient la cause lointaine de l'ensemble de l'épisode de Clavegris; la tempête gêne les déplacements des personnages ou réorientent leur itinéraire, et cela dès le prologue où elle est

lyeppart et le corps d'un moult grant dragon, mais vers les cuisses estoit a maniere de serpent. 11 Quant mengier voloit poisson, il se bouttoit en la mer et quant char voloit avoir, il se mettoit en Albanye, ou il ne failloit point que tous les jours n'euist ung homme ou ung moutton. $12 \mathrm{Se}$ aulcunement on lui defailloit de luy livrer sa porcion, il n'arestoit point jusques il euist devouré douze ou treize* homez, par coy tant en estoit la terre apovrye autour de la citey (XXI, 8-12)

${ }^{36} 7 \mathrm{Je}$ voel bien que tu saches que je suis dame et roÿne de l'Isle Chelee, qui pour l'amour de toy ay passé la mer, ja n'y a il plus d'oir que moy. 8 Se tu t'en voelz venir avoec moy demourer, demain te feray roy et je seray roÿne. (XXVIII, 7-8)

${ }^{37}$ Il sot tous les ars et syences bonnes et males que homme pot savoir. Il fist moult de maulx en son temps. Quant il voloit aller en l'Isle Celee pour rober et pour pillier, par ses enchantemens faisoit les nefs plongier et perir en la mer. (LII, 30).

${ }^{38}$ Op. cit., p. 339. 
l'instrument de la puissance divine : Dieu, la mer et le vent conduisent les Picards en la chité de Sallenicque (Pr., 2-3) et provoquent la découverte du livre-source et donc l'écriture ellemême : le saint pèlerinage devient une quête du passé, à la gloire de la chevalerye grigoise qui jadis fu moult hault eslevee par tous rengnes.

$$
* * *
$$

Le Roman de Florimont est donc un bon exemple de la perception du voyage maritime méditerranéen dans les romans du cycle Wavrin; plus qu'une description précise des éléments matériels, relativement peu nombreux, le voyage est d'abord perçu comme expédition militaire dans la construction du royaume idéal qui, alors même que l'orient vit sous la menace perpétuelle des conquêtes ottomanes, élimine les agresseurs, pirates et Sarrasins et unit harmonieusement les peuples sous la domination d'un prince idéalisé, la fleur de la chevalerie grygoise, modèle et référence pour les principautés occidentales.

Marie-Madeleine Castellani

ALITHILA 